\title{
Stable, Doubly Heavy Tetraquark Mesons
}

Chris Quigg

Fermilab \& Nikhef

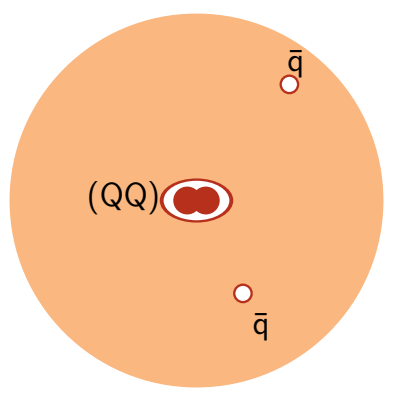

Nico van Kampen Colloquium · Utrecht · 4 April 2018 - DOI: 10.5281/zenodo.1213296

Estia Eichten \& CQ, PRL 119, 202002 (2017) / arXiv:1707.09575 


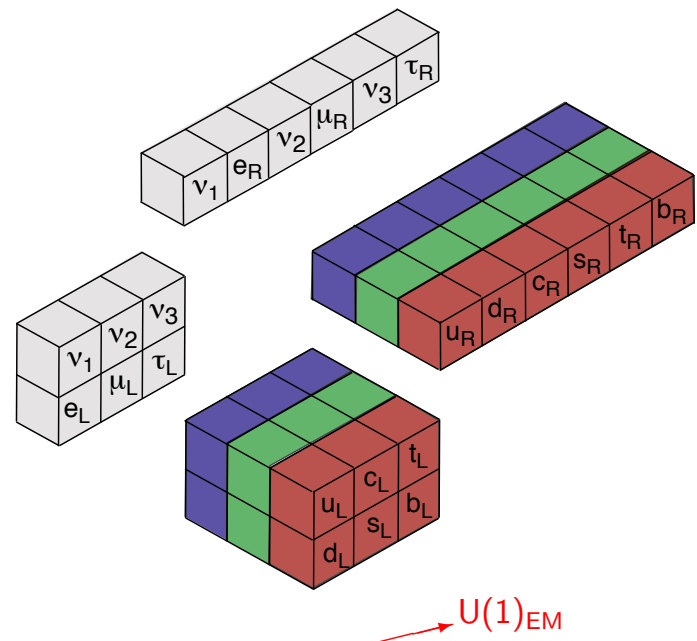

$\mathrm{SU}(3)_{c} \otimes S U(2)_{t} \otimes U(1)_{Y}$ gauge symmetries 


\section{Ancient History: Hadron Spectroscopy \& Flavor SU(3)}

Gell-Mann, "The Eightfold Way" \& Ne'eman, Nucl. Phys. 26, 229 (1961): SU(3) classification symmetry
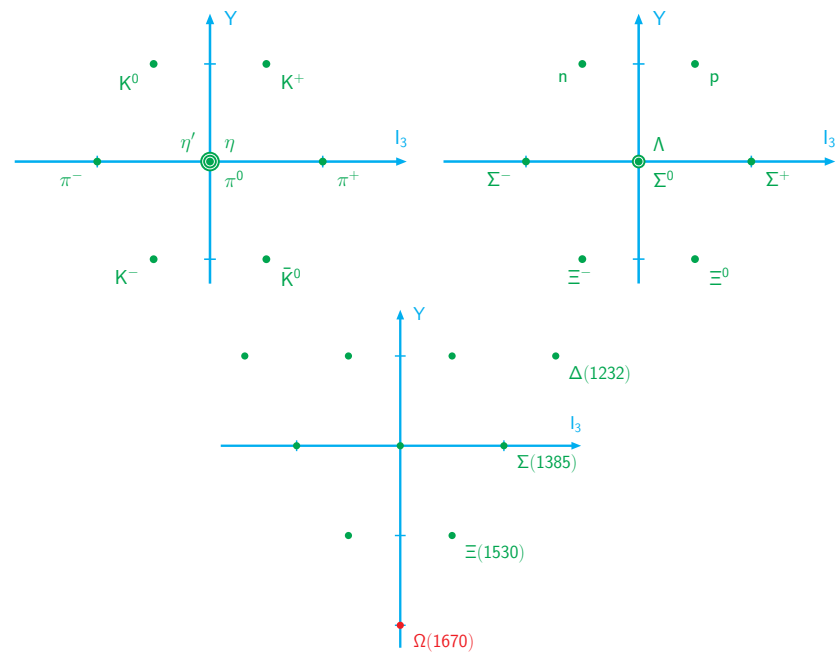

The Octet Model and its Clebsch-Gordan Coefficients

J. J. DE Swart*
CERN, Geneva

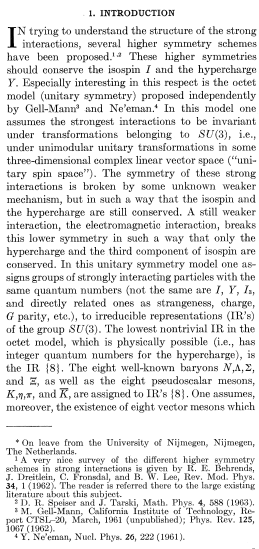

belong to such a representation. Perhaps the mesons which $K^{*}$ constitute this octet. A difficulty here resonances, one tat $730 \mathrm{MeV}$ and the other at 888 it The next higher IR cun contain 10 particles. It is suggested that the familini $(3,3)$ pion-nucleon resonance, the $Y_{t}^{*}(1385 \mathrm{MeV})$, the recently dis till unknown berron $\Omega^{-}(Y=-2, I=0,=0$. MeV) belong to this IR $\{10\}$. A discovery of this 8 would be a grent triumph for this octet model. Okubo $0^{10}$ has derived a mass formula for the different (IR (8) this formula reduces to a mass ration setween the different members. This mass relation is very well satisfied for the baryons and for the pseudoscalar mesons. However, for the vector

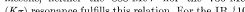
this mass formula is again very well satiafied. Coleman and Glashow" have given a relation connecting the electromagnetic mass difierences within the The main purpoes of this poper is to derive the

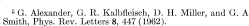

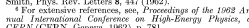

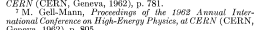

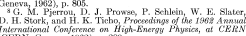

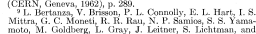

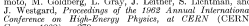

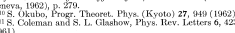




\section{Ancient History: Hadron Spectroscopy \& Quarks}

1964: Gell-Mann ("quarks") and Zweig ("aces") noticed that observed multiplets could be constructed from elementary spin- $\frac{1}{2}$ flavor triplets-isospin doublet $(u, d)$ and strange isospin singlet $s$, according to the rules meson $=q \bar{q}$ and baryon $=q q q$.

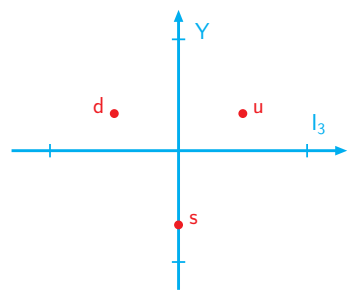

SU(3) flavor algebra:

$$
\mathbf{3} \otimes \overline{\mathbf{3}}=\mathbf{1} \oplus \mathbf{8}
$$

$\mathbf{3} \otimes \mathbf{3} \otimes \mathbf{3}=\mathbf{1} \oplus \mathbf{8} \oplus \mathbf{8} \oplus \mathbf{1 0}$

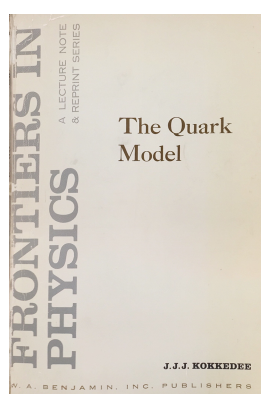

"Of course, the whole quark idea is ill-founded. So far, quarks have escaped detection. This fact could simply be taken to mean that they are extremely massive and therefore difficult to produce, but it could also be an indication that quarks cannot exist as individual particles but, like phonons in a crystal, can have meaning only inside the hadrons. In either case, nevertheless, the dynamical system of such quarks binding together to give the observed hadrons that has the properties demanded by the applications, is very difficult to understand in terms of conventional concepts. The quark model should, therefore, at least for the moment, not be taken for more than what it is, namely the tentative and simplistic expression of an as yet obscure dynamics underlying the hadronic world." 


\section{Establishing the quark paradigm: pointlike constituents}

Bjorken scaling in deeply inelastic scattering

$$
R \equiv \sigma\left(e^{+} e^{-} \rightarrow \text { hadrons }\right) / \sigma\left(e^{+} e^{-} \rightarrow \mu^{+} \mu^{-}\right)
$$
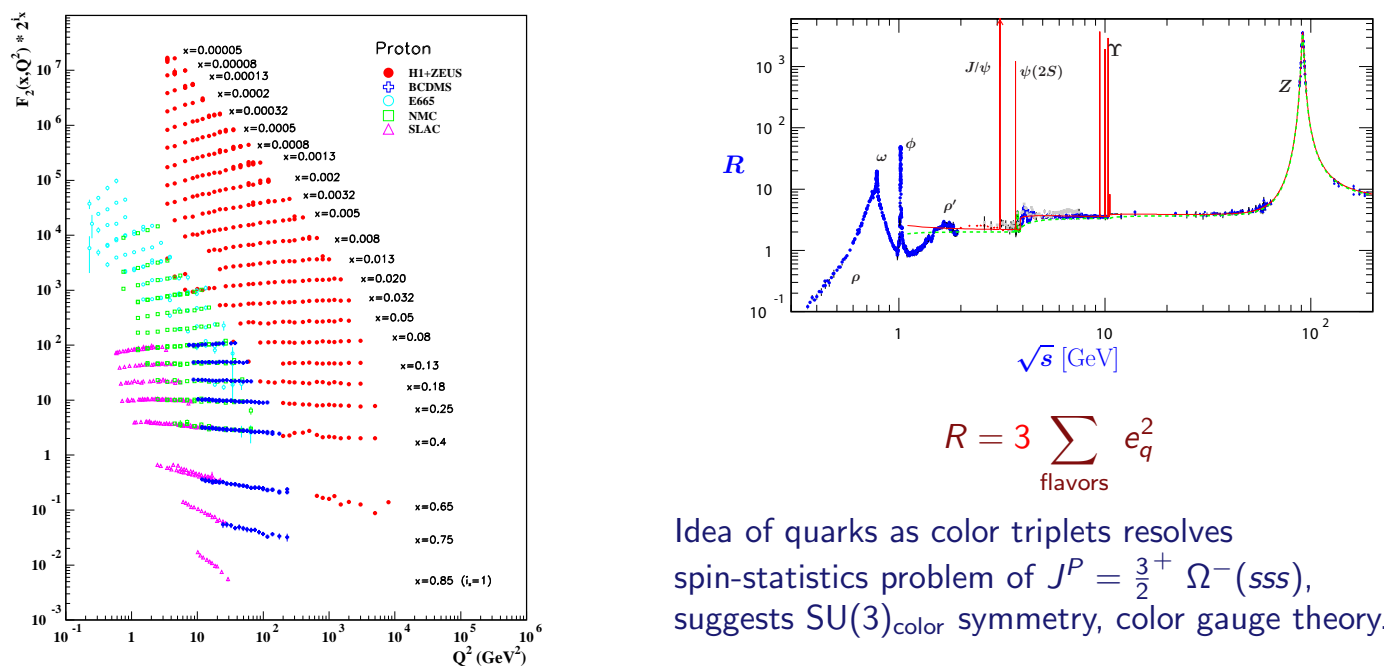

Idea of quarks as color triplets resolves spin-statistics problem of $J^{P}=\frac{3}{2}^{+} \Omega^{-}$(sss), suggests $\mathrm{SU}(3)_{\text {color }}$ symmetry, color gauge theory. 


\section{Color confinement, perturbation theory, concrete quarks}

Asymptotic freedom

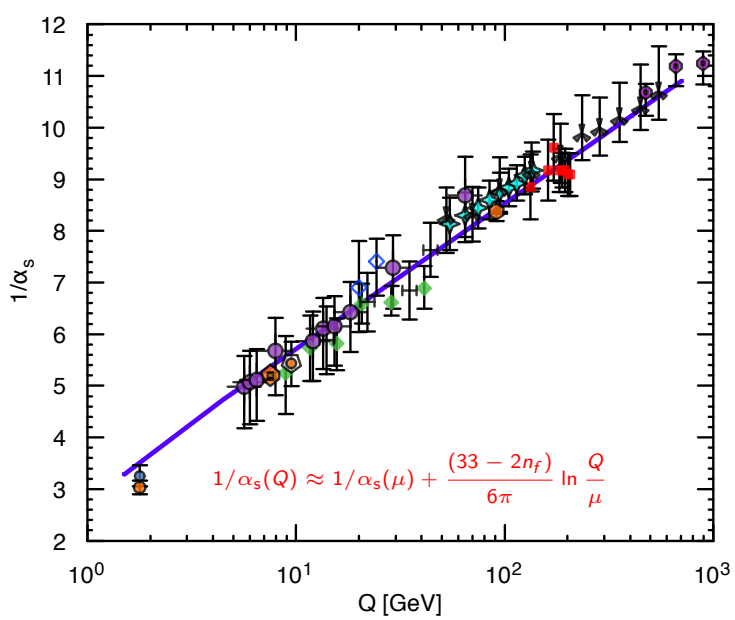

Spectrum of charmonium $(c \bar{c})$ states

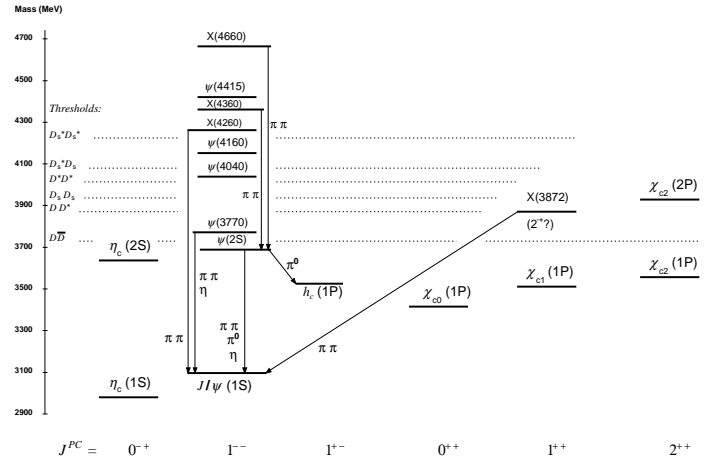




\section{Heavy-flavor hadrons}

Spectrum of bottomonium $(b \bar{b})$ states

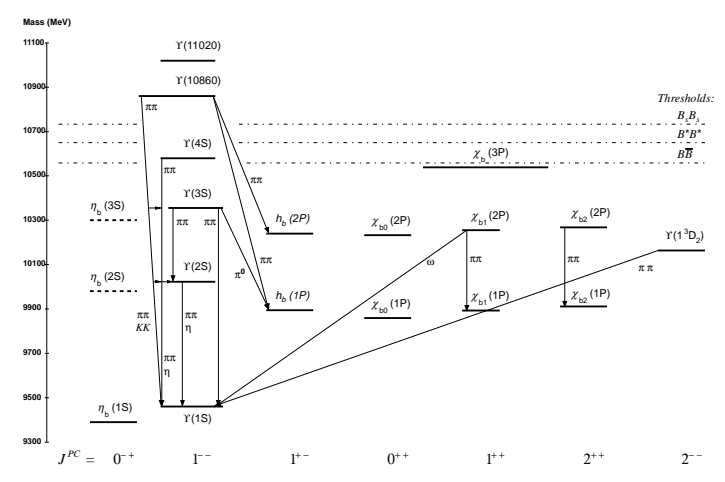

$t \bar{t}$ in the $\mathrm{D} \varnothing$ experiment at Fermilab

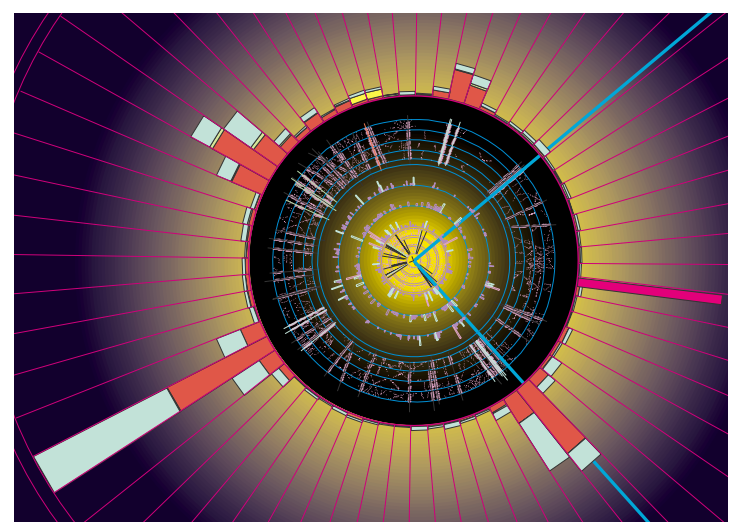




\section{Hadron masses from Lattice $Q C D$}

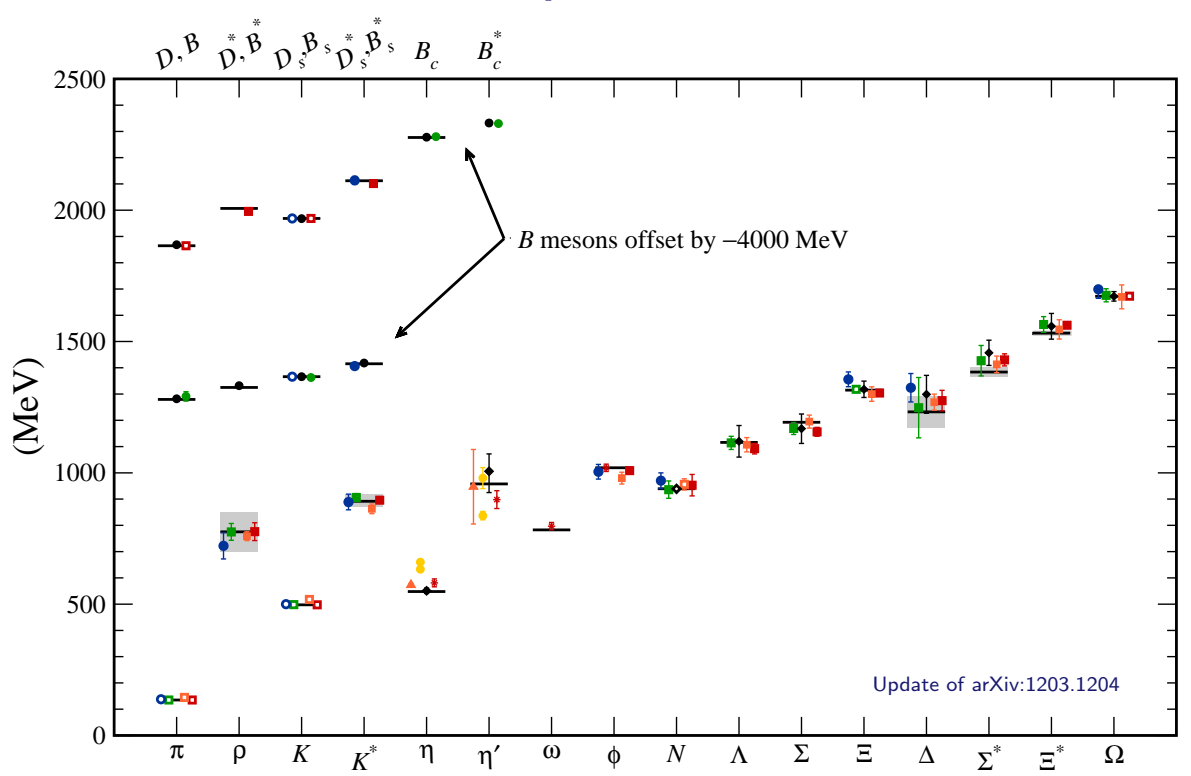




\section{Recent Prehistory (2002-2003) ...}

BELLE observes $\eta_{c}^{\prime}(3654)$ in $B \rightarrow K K_{s} K^{-} \pi^{+}$decays (now $\eta_{c}^{\prime}(3636)$ ).

ELQ advocate $B$-meson gateways to missing charmonium levels

$$
h_{c}\left(1^{1} \mathrm{P}_{1}\right), \eta_{c 2}\left(1^{1} \mathrm{D}_{2}\right) \text {, and } \psi_{2}\left(1^{3} \mathrm{D}_{2}\right)
$$

BELLE observes $X(3872)$ in $B^{ \pm} \rightarrow K^{ \pm} \pi^{+} \pi^{-} J / \psi$ decays $\left(D^{0} \bar{D}^{* 0}\right.$ mass!)
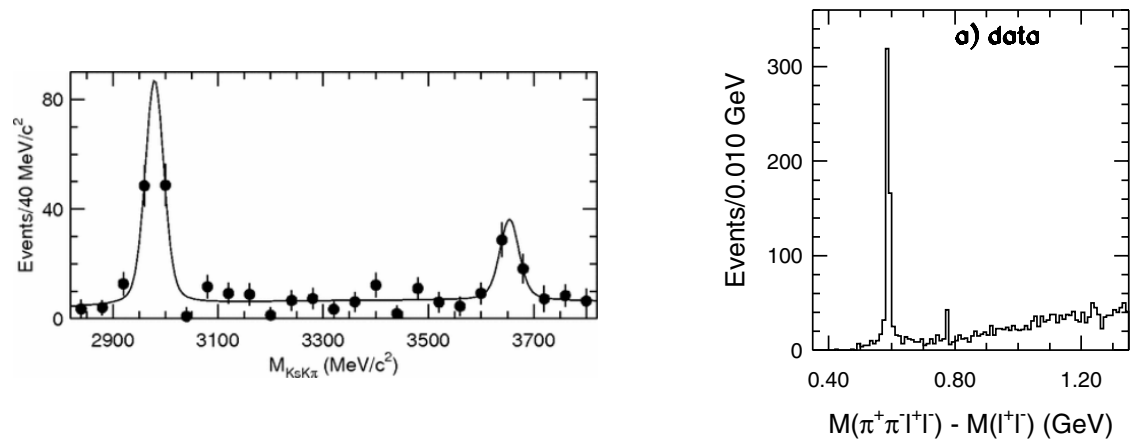
$X(3872) \leadsto$ Renaissance in hadron spectroscopy ... $X(3872) \neq \psi_{2}\left(1^{3} D_{2}\right): J^{P C}=1^{++}$

$c \bar{c}$ state modified by coupling with open channels?

Threshold "cusp" phenomenon?

$D-\bar{D}^{*}$ molecule? / hadrocharmonium

Tetraquark (diquark-antidiquark) meson?

QM superposition of several Fock states

Isospin violation likely

Other new states invite hybrid $(c \bar{c} g)$ interpretations, etc.

Clarity from change with heavy-quark mass $(c \bar{c}),(b \bar{c}),(b \bar{b})$ ? 


\section{$X(3872) \leadsto$ Renaissance in hadron spectroscopy ...}

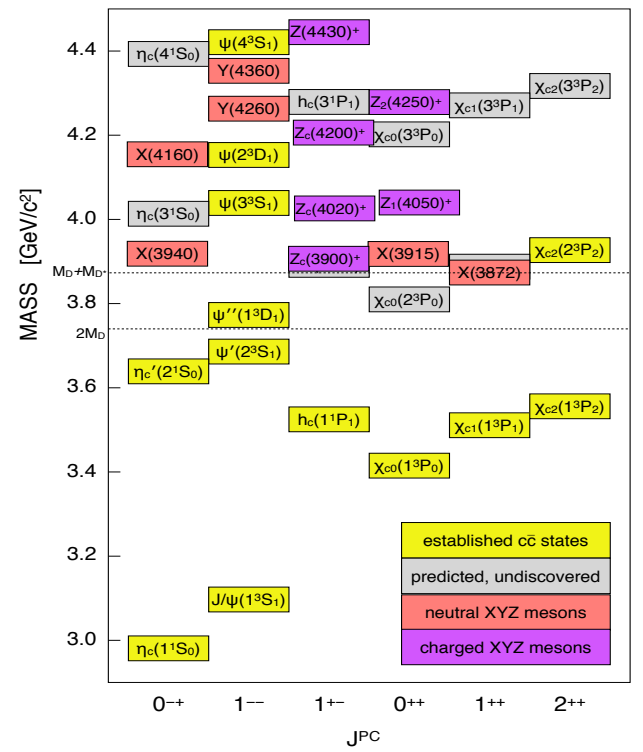

S. L. Olsen, Front. Phys. (Beijing) 10, 121 (2015) [arXiv:1411.7738].

R. F. Lebed, R. E. Mitchell, E. S. Swanson, Prog. Part. Nucl. Phys. 93, 143 (2017) [arXiv:1610.04528].

A. Esposito, A. Pilloni, A. D. Polosa, Phys. Rept. 668, 1 (2016) [arXiv:1611.07920].

A. Ali, J. S. Lange, S. Stone, Prog. Part. Nucl. Phys. 97, 123 (2017) [arXiv:1706.00610].

S. L. Olsen, T. Skwarnicki and D. Zieminska, Rev. Mod. Phys. 90, 015003 (2018) [arXiv:1708.04012].

F.-K. Guo, C. Hanhart, Ulf-G. Meißner, Q. Wang, Q. Zhao, and B.-S. Zou. Rev. Mod. Phys. 90, 015004 (2018). 


\section{Charged states invite tetraquark interpretations}

Lo-o-o-o-ng history, dating to foundational papers of the quark model

G. Zweig, "An SU(3) model for strong interaction symmetry and its breaking," CERN-TH-401 (1964); "An SU(3) model for strong interaction symmetry and its breaking. 2," CERN-TH-412 (1964).

M. Gell-Mann, "A schematic model of baryons and mesons," Phys. Lett. 8, 214-215 (1964).

Application to (light-)meson spectroscopy: broad scalars $a_{0}(980), f_{0}(980)$ R. L. Jaffe, "Multi-Quark Hadrons. 1. The Phenomenology of $\left(q^{2} \bar{q}^{2}\right)$ Mesons," Phys. Rev. D 15, 267 (1977); "Multi-Quark Hadrons. 2. Methods," Phys. Rev. D 15, 281 (1977).

Tetraquark interpretations of $X Y Z$ complicated by many thresholds Tetraquark advocate: L. Maiani, "Exotic Hadrons," CERN Heavy-hadron Spectroscopy, July 2017

Can we unambiguously demonstrate the reality of tetraquarks? 
Heavy-quark symmetry implies stable heavy tetraquark mesons $Q_{i} Q_{j} \bar{q}_{k} \bar{q}_{l}$ In the limit of very heavy quarks $Q$, novel narrow doubly heavy tetraquark states (DHTQ) must exist.

$H Q S$ relates DHTQ mass to masses of a doubly heavy baryon, heavy-light baryon, and heavy-light meson.

The lightest double-beauty states composed of bbū $\bar{d}, b b \bar{u} \bar{s}$, and $b b \bar{d} \bar{s}$ will likely be stable against strong decays.

Heavier $b b \bar{q}_{k} \bar{q}_{l}$ states, $c c \bar{q}_{k} \bar{q}_{l}$ states, and mixed $b c \bar{q}_{k} \bar{q}_{l}$ states, will likely dissociate into pairs of heavy-light mesons. Some might be seen as "double-flavor" resonances near threshold.

Observing a weakly decaying double-beauty state would establish the existence of tetraquarks and illuminate the role of

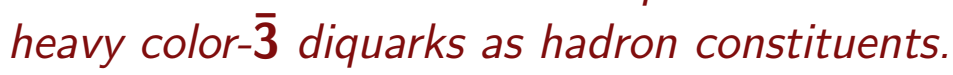




\section{When tetraquarks resemble the helium atom ...}

Factorized system: separate dynamics for compact "nucleus," light quarks

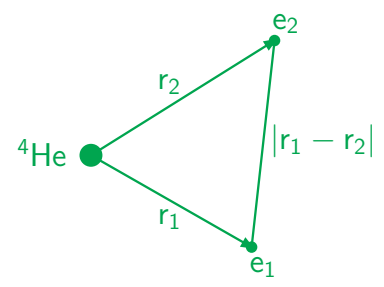

(Attractive, repulsive) one-gluon exchange for $(Q Q)$ in color- $(\overline{\mathbf{3}}, \mathbf{6})$ $\overline{3}$ half strength of $Q \bar{Q}$ attraction in color-1 also for string tension [Nakamura \& Saito]

In heavy limit, idealize a stationary, structureless (color) charge 


\section{Stability in the heavy-quark limit}

1) Dissociation into two heavy-light mesons is kinematically forbidden.

$$
\begin{aligned}
& \mathcal{Q} \equiv m\left(Q_{i} Q_{j} \bar{q}_{k} \bar{q}_{l}\right)-\left[m\left(Q_{i} \bar{q}_{k}\right)+m\left(Q_{j} \bar{q}_{l}\right)\right]= \\
& \underbrace{\Delta\left(q_{k}, q_{l}\right)}_{\text {light d.o.f. }}-\frac{1}{2}\left(\frac{2}{3} \alpha_{s}\right)^{2}\left[1+O\left(v^{2}\right)\right] \bar{M}+O(1 / \bar{M}),
\end{aligned}
$$

$\bar{M} \equiv\left(1 / m_{Q_{i}}+1 / m_{Q_{j}}\right)^{-1}:$ reduced mass of $Q_{i}$ and $Q_{j}$ $\Delta\left(q_{k}, q_{l}\right) \stackrel{\bar{M} \rightarrow \infty}{\longrightarrow}$ independent of heavy-quark masses

For large enough $\bar{M}, Q Q$ Coulomb binding dominates, $\mathcal{Q}<0$ 
Stability in the heavy-quark limit

2) Decay to doubly heavy baryon and light antibaryon?

$$
\left(Q_{i} Q_{j} \bar{q}_{k} \bar{q}_{l}\right) \rightarrow\left(Q_{i} Q_{j} q_{m}\right)+\left(\bar{q}_{k} \bar{q}_{l} \bar{q}_{m}\right)
$$

Core $Q_{i} Q_{j}$ is color $\overline{\mathbf{3}}$, same as $\bar{Q}_{x}$. Up to contributions from $Q$ motion and spin interactions,

$$
m\left(Q_{i} Q_{j} \bar{q}_{k} \bar{q}_{l}\right)-m\left(Q_{i} Q_{j} q_{m}\right)=m\left(Q_{x} q_{k} q_{l}\right)-m\left(Q_{x} \bar{q}_{m}\right)
$$

(spin configurations matter)

RHS has generic form $\Delta_{0}+\Delta_{1} / M_{Q_{x}}$

Using $m\left(\Lambda_{c}\right)-m(D)=416.87 \mathrm{MeV}$ and $m\left(\Lambda_{b}\right)-m(B)=340.26 \mathrm{MeV}$, we estimate $\Delta_{0} \approx 330 \mathrm{MeV}$ (asymptotic mass difference).

$$
\text { All }<m(\bar{p})=938 \mathrm{MeV}
$$


No open strong decay channels in the heavy-quark limit!
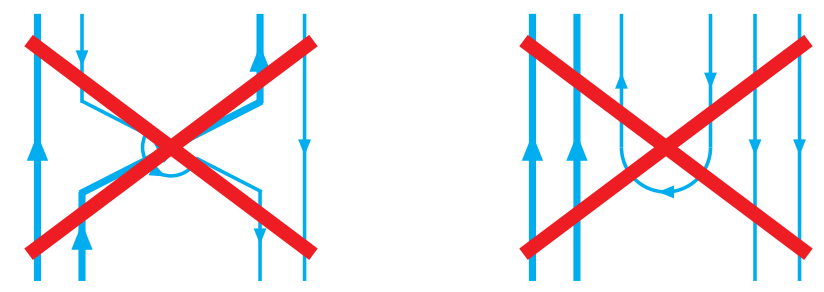

As $\bar{M} \rightarrow \infty$, stable $Q_{i} Q_{j} \bar{q}_{k} \bar{q}_{l}$ mesons must exist

Implications for the real world? 
Does a tiny quasistatic diquark core make sense in our world?

At large $Q_{i}-Q_{j}$ separations, $\bar{q}_{k} \bar{q}_{l}$ cloud screens $Q_{i} Q_{j}$ interaction

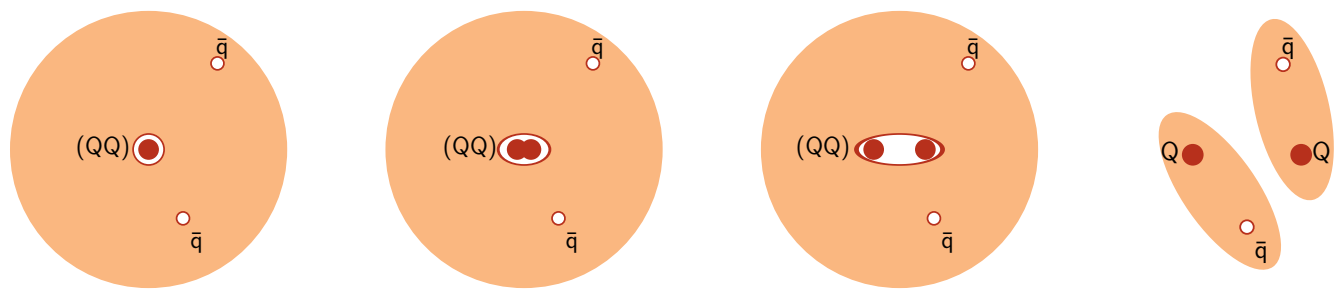

Growing separation alters $\overline{\mathbf{3}}, \mathbf{6} \mathrm{mix} \leadsto$ division into heavy-light mesons

In a half-strength Cornell potential, rms core radii are small on tetraquark scale: $\left\langle r^{2}\right\rangle^{1 / 2}=0.28 \mathrm{fm}(c c) ; 0.24 \mathrm{fm}(b c) ; 0.19 \mathrm{fm}(b b)$.

(lattice, too)

$\therefore$ core-plus-light (anti)quarks idealization should be reliable. 
Mass estimates (beyond the heavy-quark limit ... )

Use heavy-quark-symmetry relations,

$$
\begin{aligned}
m\left(\left\{Q_{i} Q_{j}\right\}\left\{\bar{q}_{k} \bar{q}_{l}\right\}\right)-m\left(\left\{Q_{i} Q_{j}\right\} q_{y}\right) & =m\left(Q_{x}\left\{q_{k} q_{l}\right\}\right)-m\left(Q_{x} \bar{q}_{y}\right) \\
m\left(\left\{Q_{i} Q_{j}\right\}\left[\bar{q}_{k} \bar{q}_{l}\right]\right)-m\left(\left\{Q_{i} Q_{j}\right\} q_{y}\right) & =m\left(Q_{x}\left[q_{k} q_{l}\right]\right)-m\left(Q_{x} \bar{q}_{y}\right) \\
m\left(\left[Q_{i} Q_{j}\right]\left\{\bar{q}_{k} \bar{q}_{l}\right\}\right)-m\left(\left[Q_{i} Q_{j}\right] q_{y}\right) & =m\left(Q_{x}\left\{q_{k} q_{l}\right\}\right)-m\left(Q_{x} \bar{q}_{y}\right) \\
m\left(\left[Q_{i} Q_{j}\right]\left[\bar{q}_{k} \bar{q}_{l}\right]\right)-m\left(\left[Q_{i} Q_{j}\right] q_{y}\right) & =m\left(Q_{x}\left[q_{k} q_{l}\right]\right)-m\left(Q_{x} \bar{q}_{y}\right) . \\
& \\
+ \text { finite-mass corrections, } \delta m & =\mathcal{S} \frac{\vec{S} \cdot \overrightarrow{j_{\ell}}}{2 \mathcal{M}}+\frac{\mathcal{K}}{2 \mathcal{M}}
\end{aligned}
$$

(hyperfine + light d.o.f.)

to estimate $Q_{i} Q_{j} \bar{q}_{k} \bar{q}_{l}$ masses 
Masses, etc., for ground-state hadrons containing heavy quarks

State

$D^{(*)}(c \bar{d}) \quad \frac{1}{2}$

$D_{s}^{(*)}(c \bar{s}) \quad \frac{1}{2}$

$\Lambda_{c}(\text { cud })_{\overline{3}} \quad 0$

$\Sigma_{c}(\text { cud })_{6} \quad 1$

$\Xi_{c}(\text { cus })_{\overline{3}} \quad 0$

$\Xi_{c}^{\prime}(\text { cus })_{6} \quad 1$

$\Omega_{c}(\text { css })_{6} \quad 1$

$\Xi_{c c}(c c u)_{\overline{3}} \quad 0$

$B^{(*)}(b \bar{d}) \quad \frac{1}{2}$

$B_{s}^{(*)}(b \bar{s}) \quad \frac{1}{2}$

$\Lambda_{b}(\text { bud })_{\overline{3}} \quad 0$

$\Sigma_{b}(\text { bud })_{6} \quad 1$

$\Xi_{b}(b d s)_{\overline{3}} \quad 0$

$\Xi_{b}^{\prime}(b d s)_{6} \quad 1$

$\Omega_{b}$ (bss) 1

$B_{c}(b \bar{c})$
Mass $\left(j_{\ell}+\frac{1}{2}\right)$

2010.26

2112.1

2286.46

2518.41

2467.87

2645.53

2765.9

3621.40

5324.65

5415.4

5619.58

5832.1

5794.5

5955.33

6329 Mass $\left(j_{\ell}-\frac{1}{2}\right)$

Centroid

1869.59

1968.28

$-$

2453.97

2577.4

2695.2

$-$

5279.32

5366.89

$-$

5811.3

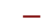

5935.02

6046.1

6274.9
1975.09

2076.15

2496.93

2622.82

2742.33

5313.32

5403.3

5825.2

5948.56

6315.4
140.7

143.8

64.44

68.13

70.7

$-$

45.33

48.5

$-$

20.8

$-$

20.31

54
0.128

0.436

0.446

0.132

0.141

0.146

0.427

0.459

0.131

0.340 


\section{Kinetic-energy shift differs in $Q \bar{q}$ mesons and $Q q q$ baryons ...}

Consider $\delta \mathcal{K} \equiv \mathcal{K}_{(u d)}-\mathcal{K}_{d}$ :

$$
\begin{gathered}
{\left[m\left((c u d)_{\overline{3}}\right)-m(c \bar{d})\right]-\left[m\left((\text { bud })_{\overline{3}}\right)-m(b \bar{d})\right]} \\
=\delta \mathcal{K}\left(\frac{1}{2 m_{c}}-\frac{1}{2 m_{b}}\right)=5.11 \mathrm{MeV} \\
\sim \delta \mathcal{K}=0.0235 \mathrm{GeV}^{2} \\
m(\{c c\}(\bar{u} \bar{d}))-m(\{c c\} d): \quad \frac{\delta \mathcal{K}}{4 m_{c}}=2.80 \mathrm{MeV} \\
m((b c)(\bar{u} \bar{d}))-m(\{b c\} d): \quad \frac{\delta \mathcal{K}}{2\left(m_{c}+m_{b}\right)}=1.87 \mathrm{MeV} \\
m(\{b b\}(\bar{u} \bar{d}))-m(\{b b\} d): \quad \frac{\delta \mathcal{K}}{4 m_{b}}=1.24 \mathrm{MeV}
\end{gathered}
$$

Small! (only slightly larger than isospin-breaking effects we neglect) 
Estimating ground-state tetraquark masses

RHS of

$$
m\left(Q_{i} Q_{j} \bar{q}_{k} \bar{q}_{l}\right)-m\left(Q_{i} Q_{j} q_{m}\right)=m\left(Q_{x} q_{k} q_{l}\right)-m\left(Q_{x} \bar{q}_{m}\right)
$$

is determined from data

One doubly heavy baryon observed, $\Xi_{c c}$; others from model calculations ${ }^{\star}$

$$
\mathrm{LHCb}: M\left(\Xi_{c c}^{++}\right)=3621.40 \pm 0.78 \mathrm{MeV}
$$

*We adopt Karliner \& Rosner, PRD 90, 094007 (2014)

Strong decays $\left(Q_{i} Q_{j} \bar{q}_{k} \bar{q}_{l}\right) \not \rightarrow\left(Q_{i} Q_{j} q_{m}\right)+\left(\bar{q}_{k} \bar{q}_{l} \bar{q}_{m}\right) \forall$ ground states

Must consider decays to pairs of heavy-light mesons case-by-case 
Expectations for ground-state tetraquark masses, in $\mathrm{MeV}$

\begin{tabular}{lcccc} 
State & $J^{P}$ & $m\left(Q_{i} Q_{j} \bar{q}_{k} \bar{q}_{l}\right)$ & Decay Channel & $\mathcal{Q}[\mathrm{MeV}]$ \\
\hline$\{c c\}[\bar{u} \bar{d}]$ & $1^{+}$ & 3978 & $D^{+} D^{* 0} 3876$ & 102 \\
$\{c c\}\left[\bar{q}_{k} \bar{s}\right]$ & $1^{+}$ & 4156 & $D^{+} D_{s}^{*+} 3977$ & 179 \\
$\{c c\}\left\{\bar{q}_{k} \bar{q}_{l}\right\}$ & $0^{+}, 1^{+}, 2^{+}$ & $4146,4167,4210$ & $D^{+} D^{0}, D^{+} D^{* 0} 3734,3876$ & $412,292,476$ \\
{$[b c][\bar{u} \bar{d}]$} & $0^{+}$ & 7229 & $B^{-} D^{+} / B^{0} D^{0} 7146$ & 83 \\
{$[b c]\left[\bar{q}_{k} \bar{s}\right]$} & $0^{+}$ & 7406 & $B_{s} D 7236$ & 170 \\
{$[b c]\left\{\bar{q}_{k} \bar{q}_{l}\right\}$} & $1^{+}$ & 7439 & $B^{*} D / B D^{*} 7190 / 7290$ & 249 \\
$\{b c\}[\bar{u} \bar{d}]$ & $1^{+}$ & 7272 & $B^{*} D / B D^{*} 7190 / 7290$ & 82 \\
$\{b c\}\left[\bar{q}_{k} \bar{s}\right]$ & $1^{+}$ & 7445 & $B_{s}^{*} 7282$ & 163 \\
$\{b c\}\left\{\bar{q}_{k} \bar{q}_{l}\right\}$ & $0^{+}, 1^{+}, 2^{+}$ & $7461,7472,7493$ & $B D / B^{*} D 7146 / 7190$ & $317,282,349$ \\
$\{b b\}[\bar{u} \bar{d}]$ & $1^{+}$ & 10482 & $B^{-} \bar{B}^{* 0} 10603$ & -121 \\
$\{b b\}\left[\bar{q}_{k} \bar{s}\right]$ & $1^{+}$ & 10643 & $\bar{B}_{s}^{*} / \bar{B}_{s} \bar{B}^{*} 10695 / 10691$ & -48 \\
$\{b b\}\left\{\bar{q}_{k} \bar{q}_{l}\right\}$ & $0^{+}, 1^{+}, 2^{+}$ & $10674,10681,10695$ & $B^{-} B^{0}, B^{-} B^{* 0} 10559,10603$ & $115,78,136$ \\
\hline
\end{tabular}

Cf. M. Karliner \& J. L. Rosner model, Phys. Rev. Lett. 119, 202001 (2017) [arXiv:1707.07666]. Estimate deeper binding, so additional $b c$ and $c c$ candidates. 
Real-world candidates for stable tetraquarks

$J^{P}=1^{+}\{b b\}[\bar{u} \bar{d}]$ meson, bound by $121 \mathrm{MeV}$

(77 MeV below $\left.B^{-} \bar{B}^{0} \gamma\right)$

$$
\mathcal{T}_{[\bar{u} \bar{d}]}^{\{b b\}}(10482)^{-} \rightarrow \Xi_{b c}^{0} \bar{p}, B^{-} D^{+} \pi^{-}, \text {and }
$$

$$
\underbrace{B^{-} D^{+} \ell^{-} \bar{\nu}}_{\text {manifestly weak! }}
$$

$J^{P}=1^{+}\{b b\}[\bar{u} \bar{s}]$ and $\{b b\}[\bar{d} \bar{s}]$ mesons, bound by $48 \mathrm{MeV}$

(3 $\mathrm{MeV}$ below $B B_{s} \gamma$ )

$$
\mathcal{T}_{[\bar{u} \bar{s}]}^{\{b b\}}(10643)^{-} \rightarrow \Xi_{b c}^{0} \bar{\Sigma}^{-} \quad \mathcal{T}_{[\bar{d} \bar{s}]}^{\{b b\}}(10643)^{0} \rightarrow \Xi_{b c}^{0}\left(\bar{\Lambda}, \bar{\Sigma}^{0}\right)
$$

SELEX $M\left(\Xi_{c c}^{+}\right)=3519 \mathrm{MeV} \sim m(\{c c\}[\bar{u} \bar{d}])=3876 \mathrm{MeV}$, at threshold for dissociation into a heavy-light pseudoscalar and heavy-light vector. Signatures for weak decay would include $D^{+} K^{-} \ell^{+} \nu$ and $\Xi_{c}^{+} \bar{n} . \quad\left(D^{0} D^{+} \gamma\right.$ at $\left.3734 \mathrm{MeV}\right)$ 


\section{Lattice studies also suggest stable double-beauty tetraquarks}

P. Bicudo, K. Cichy, A. Peters and M. Wagner, PRD 93, 034501 (2016) [arXiv:1510.03441]:

$J^{P}=1^{+}\{b b\}[\bar{u} \bar{d}]$ meson, bound by $90_{-43}^{+36} \mathrm{MeV} \quad$ static $b b, m_{\pi} \approx 340 \mathrm{MeV} \ldots$

A. Francis, R. J. Hudspith, R. Lewis and K. Maltman, PRL 118, 142001 (2017) [arXiv:1607.05214]: $J^{P}=1^{+}\{b b\}[\bar{u} \bar{d}]$ meson, bound by $189 \pm 10 \mathrm{MeV}$ $b b, m_{\pi} \approx 164 \mathrm{MeV} \ldots$

$J^{P}=1^{+}\{b b\}[\bar{u} \bar{s}]$ and $\{b b\}[\bar{d} \bar{s}]$ mesons, bound by $98 \pm 7 \mathrm{MeV}$ 


\section{Unstable doubly heavy tetraquarks}

Resonances in "wrong-sign" (double flavor) combinations $D D, D B, B B$ ?

$J^{P}=1^{+} \mathcal{T}_{[\bar{d} \bar{s}]}^{\{c\}}(4156) \rightarrow D^{+} D_{s}^{*+}:$ prima facie evidence for non- $q \bar{q}$ level Double charge / double charm

(New kind of resonance: no attractive force at the meson-meson level.)

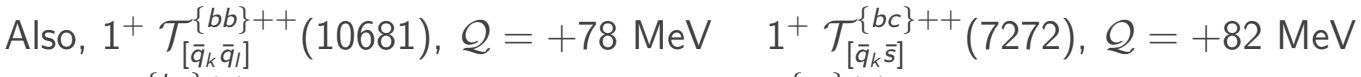

$$
\begin{aligned}
& 0^{+} \mathcal{T}_{[\bar{u} \bar{c}]}^{\{b c++}(7229), \mathcal{Q}=+83 \mathrm{MeV} \quad 1^{+} \mathcal{T}_{[\bar{u} \bar{c}]}^{\{c\}++}(3978), \mathcal{Q}=+102 \mathrm{MeV}
\end{aligned}
$$

Aside: ${ }^{3} \mathrm{D}_{3}$ and ${ }^{3} \mathrm{~F}_{4} c \bar{c}$ mesons still to be found in $D \bar{D}$, etc. 
Production of stable tetraquarks?

Undoubtedly rare! We offer no calculation, but note

- Large yield of $B_{c}$ in LHCb: $8995 \pm 103 B_{c} \rightarrow J / \psi \mu \nu_{\mu} X$ candidates in $2 \mathrm{fb}^{-1} \mathrm{pp}$ collisions at $8 \mathrm{TeV}$

- CMS observation of double- $\Upsilon$ production in 8-TeV pp collisions: $\sigma(p p \rightarrow \Upsilon \Upsilon+$ anything $)=68 \pm 15 \mathrm{pb}$

Ultimate search instrument? Future $e^{+} e^{-}$Tera- $Z$ factory

Branching fractions $Z \rightarrow b \bar{b}=15.12 \pm 0.05 \%, b \bar{b} b \bar{b}=(3.6 \pm 1.3) \times 10^{-4}$

$\leadsto$ many events containing multiple heavy quarks 


\section{Homework for experiment}

Look for double-flavor resonances near threshold.

Discover and determine masses of doubly-heavy baryons. needed to implement HQS calculation of tetraquark masses intrinsic interest in these states: comparison with heavy-light mesons, possible core excitations

$$
\text { Resolve } \Xi_{c c} \text { uncertainty (SELEX/LHCb) }
$$

Find stable tetraquarks through weak decays. Lifetime: $\sim 1 / 3$ ps ?? 


\section{Homework for theory}

Develop expectations for production.

Refine lifetime estimates for stable states.

Understand how color configurations evolve with $Q Q$ (and $\bar{q} \bar{q}$ ) masses.

Investigate stability of different body plans in the heavy-quark limit.

... up to $\left(Q_{i} Q_{j}\right)\left(Q_{k} Q_{l}\right)\left(Q_{m} Q_{n}\right): B=2$, but $Q_{p} Q_{q} Q_{r}$ color structure? 


\section{Other $Q_{i} Q_{j} \bar{q}_{k} \bar{q}_{l}$ configurations}

All quarks heavy, one-gluon exchange prevails: No stable $Q Q \bar{Q} \bar{Q}$ (equal-mass) tetraquarks in very-heavy-quark limit. Support for binding of $b b \bar{q} \bar{q}$. Study $N_{c}$ dependence.

A. Czarnecki, B. Leng, M. B. Voloshin, "Stability of tetrons," arXiv:1708.04594.

Lattice-NRQCD study of $b b \bar{b} \bar{b}$ : No tetraquark with mass below $\eta_{b} \eta_{b}$, $\eta_{b} \Upsilon, \Upsilon \Upsilon$ thresholds in $J^{P C}=0^{++}, 1^{+-}, 2^{++}$channels.

C. Hughes, E. Eichten, C. T. H. Davies, "The Search for Beauty-fully Bound Tetraquarks Using Lattice Non-Relativistic QCD," arXiv:1710.03236. 
Heavy-quark symmetry implies stable heavy tetraquark mesons $Q_{i} Q_{j} \bar{q}_{k} \bar{q}_{l}$

In the limit of very heavy quarks $Q$, novel narrow doubly heavy tetraquark states must exist.

Mass estimates lead us to expect that the $J^{P}=1^{+}\{b b\}[\bar{u} \bar{d}]$, $\{b b\}[\bar{u} \bar{s}]$, and $\{b b\}[\bar{d} \bar{s}]$ states should be exceedingly narrow, decaying only through the charged-current weak interaction

Observation would herald a new form of stable matter, in which the doubly heavy color- $\overline{3} Q_{i} Q_{j}$ diquark is a basic building block.

Unstable $Q_{i} Q_{j} \bar{q}_{k} \bar{q}_{l}$ tetraquarks with small $\mathcal{Q}$-values may be observable as resonant pairs of heavy-light mesons 